\title{
Existence of Subharmonic Solutions for a Class of Second-Order $p$-Laplacian Systems with Impulsive Effects
}

\author{
Wen-zhen Gong, ${ }^{1}$ Qiongfen Zhang, ${ }^{2}$ and X. H. Tang ${ }^{3}$ \\ ${ }^{1}$ Department of Mathematics and Computation Science, Yulin Normal University, Yulin, \\ Guangxi 537000, China \\ ${ }^{2}$ College of Science, Guilin University of Technology, Guilin, Guangxi 541004, China \\ ${ }^{3}$ School of Mathematical Sciences and Computing Technology, Central South University, Changsha, \\ Hunan 410083, China
}

Correspondence should be addressed to Qiongfen Zhang, qfzhangcsu@163.com

Received 14 April 2011; Accepted 1 July 2011

Academic Editor: Meng Fan

Copyright (c) 2012 Wen-zhen Gong et al. This is an open access article distributed under the Creative Commons Attribution License, which permits unrestricted use, distribution, and reproduction in any medium, provided the original work is properly cited.

By using minimax methods in critical point theory, a new existence theorem of infinitely many periodic solutions is obtained for a class of second-order $p$-Laplacian systems with impulsive effects. Our result generalizes many known works in the literature.

\section{Introduction}

Consider the following $p$-Laplacian system with impulsive effects:

$$
\begin{gathered}
\frac{d}{d t}\left(|\dot{u}(t)|^{p-2} \dot{u}(t)\right)-L(t)|u(t)|^{p-2} u(t)+\nabla F(t, u(t))=0, \quad \text { a.e. } t \in \mathbb{R}, \\
u(0)-u(T)=\dot{u}(0)-\dot{u}(T)=0, \\
\Delta\left(\left|\dot{u}\left(t_{j}\right)\right|^{p-2} \dot{u}\left(t_{j}\right)\right)=\left|\dot{u}\left(t_{j}^{+}\right)\right|^{p-2} \dot{u}\left(t_{j}^{+}\right)-\left|\dot{u}\left(t_{j}^{-}\right)\right|^{p-2} \dot{u}\left(t_{j}^{-}\right)=\nabla I_{j}\left(u\left(t_{j}\right)\right), \quad j=1,2, \ldots, m,
\end{gathered}
$$

where $p>1, T>0,0=t_{0}<t_{1}<t_{2}<\cdots<t_{m}<t_{m+1}=T$, and $\nabla I_{j}: \mathbb{R}^{N} \rightarrow \mathbb{R}^{N}(j=1,2, \ldots, m)$ are continuous and $F: \mathbb{R} \times \mathbb{R}^{N} \rightarrow \mathbb{R}$ is $T$-periodic in $t$ for all $u \in \mathbb{R}^{N}, \nabla F(t, u)$ is the gradient of $F(t, u)$ with respect to $u$. $L \in C\left(\mathbb{R}, \mathbb{R}^{N \times N}\right)$ is a $T$-periodic positive definite symmetric matrix. 
Throughout this paper, we always assume the following condition holds.

(A) $F(t, x)$ is measurable in $t$ for all $x \in \mathbb{R}^{N}$ and continuously differentiable in $x$ for a.e. $t \in[0, T]$, and there exist $a \in C\left(\mathbb{R}^{+}, \mathbb{R}^{+}\right), b \in L^{1}\left([0, T] ; \mathbb{R}^{+}\right)$such that

$$
|F(t, x)| \leq a(|x|) b(t), \quad|\nabla F(t, x)| \leq a(|x|) b(t)
$$

for all $x \in \mathbb{R}^{N}$ and a.e. $t \in[0, T]$.

For the sake of convenience, in the sequel, we define $B=\{1,2, \ldots, m\}$.

When $p=2, \nabla I_{j} \equiv 0, j \in B$, problem (1.1) becomes the following second-order Hamiltonian system:

$$
\ddot{u}(t)-L(t) u(t)+\nabla F(t, u(t))=0, \quad \text { a.e. } t \in \mathbb{R} \text {. }
$$

There are many papers concerning the existence of periodic solutions or homoclinic solutions for problem (1.3) by minimax methods. Here for identifying a few, we only mention [1-8].

For $\nabla I_{j} \neq 0, j \in B$, problem (1.1) involves impulsive effects. Impulsive differential equations are suitable for the mathematical simulation of evolutionary processes in which the parameters undergo relatively long periods of smooth variation followed by a short-term rapid change (that is jumps) in their values. Since these processes are subject to short-term perturbations whose duration is negligible in comparison with the duration of the processes, it is natural to suppose that these perturbations act instantaneously, that is, in the form of impulse. Processes of this type are often investigated in various fields of science and technology, for example, many biological phenomena involving thresholds, bursting rhythm models in medicine and biology, optimal control models in economics, pharmacokinetics and frequency modulated systems, and so on. For more details of impulsive differential equations, we refer the readers to the books $[9,10]$.

There are many methods for finding periodic solutions of impulsive differential equations, such as the monotone-iterative technique, a numerical-analytical method, the method of upper and lower solutions, and the method of bilateral approximations. For more information about periodic solutions of impulsive differential equations, one can refer to the papers [11-18]. However, there are few papers [19-25] concerning periodic solutions of impulsive differential equations by variational methods. So it is a novel method to employ variational methods to investigate the existence of periodic solutions for impulsive differential equations.

Motivated by the above papers, we study the existence of subharmonic solutions for problem (1.1) by applying minimax methods in critical point theory. Our result is new, which seems not to be found in the literature.

Throughout this paper, let $q \in(1,+\infty)$ satisfy $1 / p+1 / q=1$.

\section{Preliminaries}

In this section, we recall some basic facts which will be used in the proofs of our main results. In order to apply the critical point theory, we construct a variational structure. With this 
variational structure, we can reduce the problem of finding solutions of problem (1.1) to that of seeking the critical points of the corresponding functional.

Let $k$ be a positive integer and $W_{k T}^{1, p}$ the Sobolev space defined by

$W_{k T}^{1, p}=\left\{u: \mathbb{R} \rightarrow \mathbb{R}^{N} \mid u\right.$ is absolutely continuous, $\left.u(t)=u(t+k T), \dot{u} \in L^{p}\left([0, k T] ; \mathbb{R}^{N}\right)\right\}$

with the norm

$$
\|u\|=\left(\int_{0}^{k T}|u(t)|^{p} d t+\int_{0}^{k T}|\dot{u}(t)|^{p} d t\right)^{1 / p}
$$

Take $v \in W_{k T}^{1, p}$ and multiply the two sides of the equality

$$
\frac{d}{d t}\left(|\dot{u}(t)|^{p-2} \dot{u}(t)\right)-L(t)|u(t)|^{p-2} u(t)+\nabla F(t, u(t))=0
$$

by $v$ and integrate from 0 to $k T$; we have

$$
\int_{0}^{k T}\left(\left(|\dot{u}(t)|^{p-2} \dot{u}(t)\right)^{\prime}, v(t)\right) d t=\int_{0}^{k T}\left(L(t)|u(t)|^{p-2} u(t), v(t)\right) d t-\int_{0}^{k T}(\nabla F(t, u(t)), v(t)) d t
$$

Moreover, by $\dot{u}(0)=\dot{u}(T)$, one has

$$
\begin{aligned}
& \int_{0}^{k T}\left(\left(|\dot{u}(t)|^{p-2} \dot{u}(t)\right)^{\prime}, v(t)\right) d t \\
& \quad=k \int_{0}^{T}\left(\left(|\dot{u}(t)|^{p-2} \dot{u}(t)\right)^{\prime}, v(t)\right) d t \\
& \quad=k \sum_{j=0}^{m} \int_{t_{j}}^{t_{j+1}}\left(\left(|\dot{u}(t)|^{p-2} \dot{u}(t)\right)^{\prime}, v(t)\right) d t \\
& =k \sum_{j=0}^{m}\left[\left|\dot{u}\left(t_{j+1}^{-}\right)\right|^{p-2} \dot{u}\left(t_{j+1}^{-}\right) v\left(t_{j+1}^{-}\right)-\left|\dot{u}\left(t_{j}^{+}\right)\right|^{p-2} \dot{u}\left(t_{j}^{+}\right) v\left(t_{j}^{+}\right)-\int_{t_{j}}^{t_{j+1}}\left(|\dot{u}(t)|^{p-2} \dot{u}(t), \dot{v}(t)\right) d t\right] \\
& =k \sum_{j=0}^{m}\left(\left|\dot{u}\left(t_{j+1}^{-}\right)\right|^{p-2} \dot{u}\left(t_{j+1}^{-}\right) v\left(t_{j+1}^{-}\right)-\left|\dot{u}\left(t_{j}^{+}\right)\right|^{p-2} \dot{u}\left(t_{j}^{+}\right) v\left(t_{j}^{+}\right)\right)-\int_{0}^{k T}\left(|\dot{u}(t)|^{p-2} \dot{u}(t), \dot{v}(t)\right) d t \\
& =k|\dot{u}(T)|^{p-2} \dot{u}(T) v(T)-k|\dot{u}(0)|^{p-2} \dot{u}(0) v(0)-k \sum_{j=1}^{m} \nabla I_{j}\left(u\left(t_{j}\right)\right) v\left(t_{j}\right)
\end{aligned}
$$




$$
\begin{aligned}
& -\int_{0}^{k T}\left(|\dot{u}(t)|^{p-2} \dot{u}(t), \dot{v}(t)\right) d t \\
= & -k \sum_{j=1}^{m} \nabla I_{j}\left(u\left(t_{j}\right)\right) v\left(t_{j}\right)-\int_{0}^{k T}\left(|\dot{u}(t)|^{p-2} \dot{u}(t), \dot{v}(t)\right) d t .
\end{aligned}
$$

Together with (2.4), we get

$$
\begin{aligned}
& \int_{0}^{k T}\left(|\dot{u}(t)|^{p-2} \dot{u}(t), \dot{v}(t)\right) d t+k \sum_{j=1}^{m} \nabla I_{j}\left(u\left(t_{j}\right)\right) v\left(t_{j}\right)+\int_{0}^{k T}\left(L(t)|u(t)|^{p-2} u(t), v(t)\right) d t \\
& \quad=\int_{0}^{k T}(\nabla F(t, u(t)), v(t)) d t .
\end{aligned}
$$

Definition 2.1. We say that a function $u \in W_{k T}^{1, p}$ is a weak solution of problem (1.1) if the identity

$$
\begin{aligned}
& \int_{0}^{k T}\left(|\dot{u}(t)|^{p-2} \dot{u}(t), \dot{v}(t)\right) d t+k \sum_{j=1}^{m} \nabla I_{j}\left(u\left(t_{j}\right)\right) v\left(t_{j}\right)+\int_{0}^{k T}\left(L(t)|u(t)|^{p-2} u(t), v(t)\right) d t \\
& \quad=\int_{0}^{k T}(\nabla F(t, u(t)), v(t)) d t
\end{aligned}
$$

holds for any $v \in W_{k T}^{1, p}$.

Define the functional $\phi_{k}$ on $W_{k T}^{1, p}$ by

$$
\begin{aligned}
\phi_{k}(u) & =\frac{1}{p} \int_{0}^{k T}\left[|\dot{u}(t)|^{p}+\left(L(t)|u(t)|^{p-2} u(t), u(t)\right)\right] d t-\int_{0}^{k T} F(t, u(t)) d t+k \sum_{j=1}^{m} I_{j}\left(u\left(t_{j}\right)\right) \\
& =\varphi_{k}(u)+\psi_{k}(u), \quad u \in W_{k T}^{1, p},
\end{aligned}
$$

where

$$
\begin{gathered}
\varphi_{k}(u)=\frac{1}{p} \int_{0}^{k T}\left[|\dot{u}(t)|^{p}+\left(L(t)|u(t)|^{p-2} u(t), u(t)\right)\right] d t-\int_{0}^{k T} F(t, u(t)) d t \\
\psi_{k}(u)=k \sum_{j=1}^{m} I_{j}\left(u\left(t_{j}\right)\right) .
\end{gathered}
$$
$W_{k T}^{1, p}$ and

It follows from assumption (A) that the functional $\varphi_{k}$ is continuously differentiable on

$$
\left\langle\varphi_{k}^{\prime}(u), v\right\rangle=\int_{0}^{k T}\left[\left(|\dot{u}(t)|^{p-2} \dot{u}(t), \dot{v}(t)\right)+\left(L(t)|u(t)|^{p-2} u(t), v(t)\right)-(\nabla F(t, u(t)), v(t))\right] d t
$$


for $u, v \in W_{k T}^{1, p}$. By the continuity of $\nabla I_{j}, j \in B$, one has that $\psi_{k} \in\left(W_{k T}^{1, p}, \mathbb{R}\right)$. Hence, $\phi_{k}(u) \in$ $\left(W_{k T}^{1, p}, \mathbb{R}\right)$. For any $v \in W_{k T}^{1, p}$, we have

$$
\begin{aligned}
\left\langle\phi_{k}^{\prime}(u), v\right\rangle= & \int_{0}^{k T}\left[\left(|\dot{u}(t)|^{p-2} \dot{u}(t), \dot{v}(t)\right)+\left(L(t)|u(t)|^{p-2} u(t), v(t)\right)-(\nabla F(t, u(t)), v(t))\right] d t \\
& +k \sum_{j=1}^{m} \nabla I_{j}\left(u\left(t_{j}\right)\right) v\left(t_{j}\right) .
\end{aligned}
$$

By Definition 2.1, the weak solutions of problem (1.1) correspond to the critical points of the functional $\phi_{k}$.

For $u \in W_{k T}^{1, p}$, let $\bar{u}=(1 / k T) \int_{0}^{k T} u(t) d t$ and $\tilde{u}(t)=u(t)-\bar{u}$; then it follows from Proposition 1.1 in [26] that

$$
\|u\|_{\infty}:=\max _{t \in[0, k T]}|u(t)| \leq\left((k T)^{-1 / p}+(k T)^{1 / q}\right)\|u\|=d_{k}\|u\|
$$

where $d_{k}=(k T)^{-1 / p}+(k T)^{1 / q}$, and if $(1 / k T) \int_{0}^{k T} u(t) d t=0$, then

$$
\begin{gathered}
\|\tilde{u}\|_{\infty}:=\max _{t \in[0, k T]}|\tilde{u}(t)| \leq(k T)^{1 / q}\|\dot{u}\|_{L^{p}}, \\
\|\tilde{u}\|_{L^{p}}^{p} \leq(k T)^{p}\|\dot{u}\|_{L^{p}}^{p}
\end{gathered}
$$

where $1 / p+1 / q=1$. Let $\widetilde{W}_{k T}^{1, p}=\left\{u \in W_{k T}^{1, p} \mid \bar{u}=0\right\}$; then $W_{k T}^{1, p}=\widetilde{W}_{k T}^{1, p} \oplus \mathbb{R}^{N}$. We will use the following lemma to prove our main results.

Lemma 2.2 (see [27]). Let $E$ be a real Banach space with $E=X_{1} \oplus X_{2}$, where $X_{1}$ is finite dimensional. Suppose that $\varphi \in C^{1}(E, \mathbb{R})$ satisfies the (PS) condition, and

(a) there exist constants $\rho, \alpha>0$ such that $\left.\varphi\right|_{\partial B_{\rho} \cap X_{2}} \geq \alpha$, where $B_{\rho}:=\{u \in E \mid\|u\| \leq \rho\}$, and $\partial B_{\rho}$ denotes the boundary of $B_{\rho}$;

(b) there exists an $e \in \partial B_{1} \cap X_{2}$ and $L>\rho$ such that if $Q \equiv\left(\bar{B}_{L} \cap X_{1}\right) \oplus\{r e \mid 0 \leq r \leq L\}$, then $\left.\varphi\right|_{\partial Q} \leq 0$.

Then $\varphi$ possesses a critical value $c \geq \alpha$ which can be characterized as $c=\inf _{h \in \Gamma} \max _{u \in Q} \varphi(h(u))$, where $\Gamma=\{h \in C(\bar{Q}, E) \mid h=$ id on $\partial Q\}$.

It is well known that a deformation lemma can be proved with the weaker condition (C) replacing the usual (PS) condition. So Lemma 2.2 holds true under condition (C). 


\section{Main Result and Proof}

Theorem 3.1. Assume that (A) holds and $F, I_{j}$ satisfy the following conditions:

(I1) there exists $c_{j}>0$ such that

$$
0 \leq I_{j}(x) \leq \frac{{ }^{c} j}{k}|x|^{p}, \quad j \in B, \forall x \in \mathbb{R}^{N}
$$

(I2) for any $j \in B$,

$$
\nabla I_{j}(x) x \leq p I_{j}(x), \quad \forall x \in \mathbb{R}^{N}
$$

(H1) $\int_{0}^{T} F(t, x) d t \geq 0$, for all $x \in \mathbb{R}^{N}$;

(H2) $\lim _{|x| \rightarrow 0}\left(F(t, x) /|x|^{p}\right)=0$ uniformly for a.e. $t \in[0, T]$;

(H3) $\lim _{|x| \rightarrow \infty}\left(F(t, x) /|x|^{p}\right)=+\infty$ uniformly for a.e. $t \in[0, T]$;

(H4) there exists a positive constant $M$ such that $\lim \sup _{|x| \rightarrow \infty}\left(F(t, x) /|x|^{r}\right) \leq M$ uniformly for a.e. $t \in[0, T]$;

(H5) there exists $M_{1}>0$ such that $\liminf _{|x| \rightarrow \infty}((\nabla F(t, x), x)-p F(t, x)) /|x|^{\mu} \geq M_{1}$ uniformly for a.e. $t \in[0, T]$,

where $r>p$ and $\mu>r-p$. Then problem (1.1) has a sequence of distinct periodic solutions with period $k_{j} T$ satisfying $k_{j} \in \mathbb{N}$ and $k_{j} \rightarrow \infty$ as $j \rightarrow \infty$.

Remark 3.2. As far as we know, there is no paper considering subharmonic solutions of impulsive differential equations. Our result is new.

Proof. The proof is divided into three steps. In the following, $C_{i}(i=1, \ldots)$ denote different positive constants.

Step 1. The functional $\phi_{k}$ satisfies condition (C). Let $\left\{u_{n}\right\} \subset W_{k T}^{1, p}$ satisfying $(1+$ $\left.\left\|u_{n}\right\|\right)\left\|\phi_{k}^{\prime}\left(u_{n}\right)\right\| \rightarrow 0$ as $n \rightarrow \infty$ and $\phi_{k}\left(u_{n}\right)$ is bounded; then, there exists a constant $C_{1}$ such that

$$
\left|\phi_{k}\left(u_{n}\right)\right| \leq C_{1}, \quad\left(1+\left\|u_{n}\right\|\right)\left\|\phi_{k}^{\prime}\left(u_{n}\right)\right\| \leq C_{1} .
$$

From (H4), there exists $M_{2}>0$ such that

$$
F(t, x) \leq M|x|^{r} \quad \forall|x| \geq M_{2} \text {, a.e. } t \in[0, T]
$$

By assumption (A), for $|x| \leq M_{2}$, there exists $C_{2}=\max _{|x| \leq M_{2}} a(|x|)>0$ such that

$$
|F(t, x)| \leq C_{2} b(t)
$$


Journal of Applied Mathematics

which together with (3.4) implies that

$$
F(t, x) \leq M|x|^{r}+C_{2} b(t), \quad \forall x \in \mathbb{R}^{N}, \text { a.e. } t \in[0, T]
$$

By (3.3) and (3.6), we have

$$
\begin{aligned}
\phi_{k}\left(u_{n}\right)+\int_{0}^{k T} F\left(t, u_{n}\right) d t & \leq C_{1}+\int_{0}^{k T}\left(M\left|u_{n}(t)\right|^{r}+C_{2} b(t)\right) d t \\
& =C_{1}+C_{2} k\|b\|_{L^{1}}+M \int_{0}^{k T}\left|u_{n}(t)\right|^{r} d t \\
& =C_{3}+M \int_{0}^{k T}\left|u_{n}(t)\right|^{r} d t .
\end{aligned}
$$

Since $L(t)$ is continuous $T$-periodic positive definite symmetric matrix on $[0, T]$, there exist constants $c_{1}, c_{2}>0$ such that

$$
c_{1}|x|^{p} \leq\left(L(t)|x|^{p-2} x, x\right) \leq c_{2}|x|^{p}, \quad \forall x \in \mathbb{R}^{N} .
$$

It follows from (3.8) and (I1) that

$$
\begin{aligned}
\phi_{k}\left(u_{n}\right)+\int_{0}^{k T} F\left(t, u_{n}\right) d t & =\frac{1}{p} \int_{0}^{k T}\left[\left|\dot{u}_{n}(t)\right|^{p}+\left(L(t)\left|u_{n}(t)\right|^{p-2} u_{n}(t), u_{n}(t)\right)\right] d t+k \sum_{j=1}^{m} I_{j}\left(u\left(t_{j}\right)\right) \\
& \geq \frac{1}{p} \int_{0}^{k T}\left[\left|\dot{u}_{n}(t)\right|^{p}+c_{1}\left|u_{n}(t)\right|^{p}\right] d t \\
& \geq \min \left\{\frac{1}{p}, \frac{c_{1}}{p}\right\}\left\|u_{n}\right\|^{p} \\
& =C_{4}\left\|u_{n}\right\|^{p} .
\end{aligned}
$$

By (3.7) and (3.9), we get

$$
C_{4}\left\|u_{n}\right\|^{p} \leq C_{3}+M \int_{0}^{k T}\left|u_{n}(t)\right|^{r} d t
$$

From (H5), there exists $M_{3}>0$ such that

$$
(\nabla F(t, x), x)-p F(t, x) \geq M_{1}|x|^{\mu} \quad \text { for }|x| \geq M_{3} \text {, a.e. } t \in[0, T]
$$

By assumption (A), for $|x| \leq M_{3}$, there exists $C_{5}=\max _{|x| \leq M_{3}} a(|x|)>0$ such that

$$
|(\nabla F(t, x), x)-p F(t, x)| \leq C_{5}\left(p+M_{3}\right) b(t) .
$$


Thus, from (3.11) and (3.12), we have

$$
(\nabla F(t, x), x)-p F(t, x) \geq M_{1}|x|^{\mu}-M_{1} M_{3}^{\mu}-C_{5}\left(p+M_{3}\right) b(t) \quad \text { for } x \in \mathbb{R}^{N}, \text { a.e. } t \in[0, T]
$$

which together with (3.3) and (I2) implies that

$$
\begin{aligned}
(p+1) C_{1} \geq & p \phi_{k}\left(u_{n}\right)-\left\langle\phi_{k}^{\prime}\left(u_{n}\right), u_{n}\right\rangle \\
= & \int_{0}^{k T}\left[\left(\nabla F\left(t, u_{n}\right), u_{n}\right)-p F\left(t, u_{n}\right)\right] d t+p k \sum_{j=1}^{m} I_{j}\left(u_{n}\left(t_{j}\right)\right) \\
& -k \sum_{j=1}^{m} \nabla I_{j}\left(u_{n}\left(t_{j}\right)\right) u_{n}\left(t_{j}\right) \\
\geq & M_{1} \int_{0}^{k T}\left|u_{n}(t)\right|^{\mu} d t-C_{5}\left(p+M_{3}\right) \int_{0}^{k T} b(t) d t-M_{1} M_{3}^{\mu} k T \\
= & M_{1} \int_{0}^{k T}\left|u_{n}(t)\right|^{\mu} d t-C_{6} .
\end{aligned}
$$

Hence, $\int_{0}^{k T}\left|u_{n}(t)\right|^{\mu} d t$ is bounded. If $\mu>r$, we have

$$
\int_{0}^{k T}\left|u_{n}(t)\right|^{r} d t \leq(k T)^{(\mu-r) / \mu}\left(\int_{0}^{k T}\left|u_{n}(t)\right|^{\mu} d t\right)^{r / \mu}
$$

which together with (3.10) implies that $\left\|u_{n}\right\|$ is bounded. If $\mu \leq r$, then from (2.12), we get

$$
\int_{0}^{k T}\left|u_{n}(t)\right|^{r} d t \leq\left\|u_{n}\right\|_{\infty}^{r-\mu}\left(\int_{0}^{k T}\left|u_{n}(t)\right|^{\mu} d t\right)^{r / \mu} \leq d_{k}^{r-\mu}\left\|u_{n}\right\|^{r-\mu}\left(\int_{0}^{k T}\left|u_{n}(t)\right|^{\mu} d t\right)^{r / \mu}
$$

Since $\mu>r-p$, it follows from (3.10) that $\left\|u_{n}\right\|$ is bounded too. Therefore, $\left\|u_{n}\right\|$ is bounded in $W_{k T}^{1, p}$. Hence, there exists a subsequence, still denoted by $\left\{u_{n}\right\}$, such that

$$
\begin{gathered}
u_{n} \rightarrow u_{0} \quad \text { weakly in } W_{k T}^{1, p}, \\
u_{n} \longrightarrow u_{0} \quad \text { strongly in } C\left([0, k T] ; \mathbb{R}^{N}\right), \\
u_{n} \longrightarrow u_{0} \quad \text { strongly in } L^{p}\left([0, k T] ; \mathbb{R}^{N}\right) .
\end{gathered}
$$


From (2.11), we have

$$
\begin{aligned}
\left\langle\phi_{k}^{\prime}\left(u_{n}\right), u_{n}-u_{0}\right\rangle= & \int_{0}^{k T}\left[\left(\left|\dot{u}_{n}(t)\right|^{p-2} \dot{u}_{n}(t), \dot{u}_{n}(t)-\dot{u}_{0}(t)\right)+\left(L(t)\left|u_{n}(t)\right|^{p-2} u_{n}(t), u_{n}(t)-u_{0}(t)\right)\right] d t \\
& -\int_{0}^{k T}\left(\nabla F\left(t, u_{n}(t)\right), u_{n}(t)-u_{0}(t)\right) d t+k \sum_{j=1}^{m}\left(\nabla I_{j}\left(u_{n}\left(t_{j}\right)\right), u_{n}\left(t_{j}\right)-u_{0}\left(t_{j}\right)\right) .
\end{aligned}
$$

From (3.3) and (3.18), we have

$$
\left|\left\langle\phi_{k}^{\prime}\left(u_{n}\right), u_{n}-u_{0}\right\rangle\right| \leq\left\|\phi_{k}^{\prime}\left(u_{n}\right)\right\|\left\|u_{n}-u_{0}\right\| \longrightarrow 0 \quad \text { as } n \longrightarrow \infty
$$

By (3.8), we know that $c_{1} \leq\|L\| \leq c_{2}$, which together with the boundedness of $\left\{u_{n}\right\}$ and (3.19) implies that

$$
\int_{0}^{k T}\left(L(t)\left|u_{n}(t)\right|^{p-2} u_{n}(t), u_{n}(t)-u_{0}(t)\right) d t \leq\|L\|\left\|u_{n}\right\|_{L^{p}}^{p-1}\left\|u_{n}-u_{0}\right\|_{L^{p}} \longrightarrow 0 \quad \text { as } n \longrightarrow \infty .
$$

From the boundedness of $\left\{u_{n}\right\}$, the continuity of $\nabla I_{j}$, and (3.18), we have

$$
\sum_{j=1}^{m}\left(\nabla I_{j}\left(u_{n}\left(t_{j}\right)\right), u_{n}\left(t_{j}\right)-u_{0}\left(t_{j}\right)\right) \longrightarrow 0 \quad \text { as } n \longrightarrow \infty .
$$

It follows from (A), (3.18) and the boundedness of $\left\{u_{n}\right\}$ that

$$
\int_{0}^{k T}\left(\nabla F\left(t, u_{n}(t)\right), u_{n}(t)-u_{0}(t)\right) d t \longrightarrow 0 \text { as } n \longrightarrow \infty
$$

which together with (3.20), (3.21), (3.22), and (3.23) implies that

$$
\int_{0}^{k T}\left(\left|\dot{u}_{n}(t)\right|^{p-2} \dot{u}_{n}(t), \dot{u}_{n}(t)-\dot{u}_{0}(t)\right) d t \longrightarrow 0 \quad \text { as } n \longrightarrow \infty
$$

It is easy to see from the boundedness of $\left\{u_{n}\right\}$ and (3.18) that

$$
\int_{0}^{k T}\left(\left|u_{n}(t)\right|^{p-2} u_{n}(t), u_{n}(t)-u_{0}(t)\right) d t \longrightarrow 0 \quad \text { as } n \longrightarrow \infty
$$


Let $f(u)=(1 / p)\left(\int_{0}^{k T}|u(t)|^{p} d t+\int_{0}^{k T}|\dot{u}(t)|^{p} d t\right)$. Then, we have

$$
\begin{aligned}
\left\langle f^{\prime}\left(u_{n}\right), u_{n}-u_{0}\right\rangle= & \int_{0}^{k T}\left(\left|\dot{u}_{n}(t)\right|^{p-2} \dot{u}_{n}(t), \dot{u}_{n}(t)-\dot{u}_{0}(t)\right) d t \\
& +\int_{0}^{k T}\left(\left|u_{n}(t)\right|^{p-2} u_{n}(t), u_{n}(t)-u_{0}(t)\right) d t \\
\left\langle f^{\prime}\left(u_{0}\right), u_{n}-u_{0}\right\rangle= & \int_{0}^{k T}\left(\left|\dot{u}_{0}(t)\right|^{p-2} \dot{u}_{0}(t), \dot{u}_{n}(t)-\dot{u}_{0}(t)\right) d t \\
& +\int_{0}^{k T}\left(\left|u_{0}(t)\right|^{p-2} u_{0}(t), u_{n}(t)-u_{0}(t)\right) d t .
\end{aligned}
$$

It follows from (3.25) and (3.26) that

$$
\left\langle f^{\prime}\left(u_{n}\right), u_{n}-u_{0}\right\rangle \longrightarrow 0 \quad \text { as } n \longrightarrow \infty \text {. }
$$

From (3.17), we get

$$
\left\langle f^{\prime}\left(u_{0}\right), u_{n}-u_{0}\right\rangle \longrightarrow 0 \quad \text { as } n \longrightarrow \infty \text {. }
$$

By (3.27), (3.28), and Hölder's inequality, we have

$$
\begin{aligned}
& \left\langle f^{\prime}\left(u_{n}\right)-f^{\prime}\left(u_{0}\right), u_{n}-u_{0}\right\rangle \\
& =\int_{0}^{k T}\left(\left|\dot{u}_{n}(t)\right|^{p-2} \dot{u}_{n}(t), \dot{u}_{n}(t)-\dot{u}_{0}(t)\right) d t+\int_{0}^{k T}\left(\left|u_{n}(t)\right|^{p-2} u_{n}(t), u_{n}(t)-u_{0}(t)\right) d t \\
& -\int_{0}^{k T}\left(\left|\dot{u}_{0}(t)\right|^{p-2} \dot{u}_{0}(t), \dot{u}_{n}(t)-\dot{u}_{0}(t)\right) d t-\int_{0}^{k T}\left(\left|u_{0}(t)\right|^{p-2} u_{0}(t), u_{n}(t)-u_{0}(t)\right) d t \\
& =\left\|u_{n}\right\|^{p}+\left\|u_{0}\right\|^{p}-\int_{0}^{k T}\left(\left|\dot{u}_{n}(t)\right|^{p-2} \dot{u}_{n}(t), \dot{u}_{0}(t)\right) d t-\int_{0}^{k T}\left(\left|u_{n}(t)\right|^{p-2} u_{n}(t), u_{0}(t)\right) d t \\
& -\int_{0}^{k T}\left(\left|\dot{u}_{0}(t)\right|^{p-2} \dot{u}_{0}(t), \dot{u}_{n}(t)\right) d t-\int_{0}^{k T}\left(\left|u_{0}(t)\right|^{p-2} u_{0}(t), u_{n}(t)\right) d t \\
& \geq\left\|u_{n}\right\|^{p}+\left\|u_{0}\right\|^{p}-\left(\left\|u_{n}\right\|_{L^{p}}^{p-1}\left\|u_{0}\right\|_{L^{p}}+\left\|\dot{u}_{n}\right\|_{L^{p}}^{p-1}\left\|\dot{u}_{0}\right\|_{L^{p}}\right)-\left(\left\|u_{0}\right\|_{L^{p}}^{p-1}\left\|u_{n}\right\|_{L^{p}}+\left\|\dot{u}_{0}\right\|_{L^{p}}^{p-1}\left\|\dot{u}_{n}\right\|_{L^{p}}\right) \\
& \geq\left\|u_{n}\right\|^{p}+\left\|u_{0}\right\|^{p}-\left(\left\|u_{n}\right\|_{L^{p}}^{p}+\left\|\dot{u}_{n}\right\|_{L^{p}}^{p}\right)^{(p-1) / p}\left(\left\|u_{0}\right\|_{L^{p}}^{p}+\left\|\dot{u}_{0}\right\|_{L^{p}}^{p}\right)^{1 / p} \\
& -\left(\left\|u_{0}\right\|_{L^{p}}^{p}+\left\|\dot{u}_{0}\right\|_{L^{p}}^{p}\right)^{(p-1) / p}\left(\left\|u_{n}\right\|_{L^{p}}^{p}+\left\|\dot{u}_{n}\right\|_{L^{p}}^{p}\right)^{1 / p}
\end{aligned}
$$


Journal of Applied Mathematics

$$
\begin{aligned}
& =\left\|u_{n}\right\|^{p}+\left\|u_{0}\right\|^{p}-\left(\left\|u_{n}\right\|^{p-1}\left\|u_{0}\right\|+\left\|u_{0}\right\|^{p-1}\left\|u_{n}\right\|\right) \\
& =\left(\left\|u_{n}\right\|^{p-1}-\left\|u_{0}\right\|^{p-1}\right)\left(\left\|u_{n}\right\|-\left\|u_{0}\right\|\right) .
\end{aligned}
$$

Hence, from (3.29) and (3.30), we obtain

$$
0 \leq\left(\left\|u_{n}\right\|^{p-1}-\left\|u_{0}\right\|^{p-1}\right)\left(\left\|u_{n}\right\|-\left\|u_{0}\right\|\right) \leq\left\langle f^{\prime}\left(u_{n}\right)-f^{\prime}\left(u_{0}\right), u_{n}-u_{0}\right\rangle \longrightarrow 0 \quad \text { as } n \longrightarrow \infty \text {. }
$$

That is, $\left\|u_{n}\right\| \rightarrow\left\|u_{0}\right\|$ as $n \rightarrow \infty$. Since $W_{k T}^{1, p}$ has the Kadec-Klee property, we have $u_{n} \rightarrow u_{0}$ in $W_{k T}^{1, p}$. Therefore, the functional $\phi_{k}$ satisfies condition (C).

Step 2. From (H2), for any small $\varepsilon=\varepsilon(k)>0$, there exists small enough $\delta>0$ such that

$$
F(t, u) \leq \varepsilon|u|^{p} \quad \text { for }|u| \leq \delta \text {, a.e. } t \in[0, k T]
$$

For $u \in \widetilde{W}_{k T}^{1, p}$ and $\|u\|^{p}=\rho_{k}^{p}=\delta^{p} /(k T)^{p / q}$, it follows from (2.13) that

$$
\|u\|_{\infty}^{p} \leq(k T)^{p / q}\|\dot{u}\|_{L^{p}}^{p} \leq(k T)^{p / q}\|u\|^{p}=\delta^{p},
$$

which implies that $|u(t)| \leq \delta$. Then from (I1), (3.8), and (3.33), we have

$$
\begin{aligned}
\varphi_{k}(u)= & \frac{1}{p} \int_{0}^{k T}|\dot{u}(t)|^{p} d t+\frac{1}{p} \int_{0}^{k T}\left(L(t)|u(t)|^{p-2} u(t), u(t)\right) d t \\
& -\int_{0}^{k T} F(t, u) d t+k \sum_{j=1}^{m} I_{j}\left(u\left(t_{j}\right)\right) \\
\geq & \frac{1}{p} \int_{0}^{k T}|\dot{u}(t)|^{p} d t+\frac{1}{p} \int_{0}^{k T} c_{1}|u(t)|^{p} d t-\int_{0}^{k T} \varepsilon|u(t)|^{p} d t \\
\geq & \min \left\{\frac{1}{p}, \frac{c_{1}}{p}\right\}\|u\|^{p}-k T \varepsilon \delta^{p} \\
= & C_{4}\|u\|^{p}-k T \varepsilon \delta^{p} .
\end{aligned}
$$

Let $\varepsilon=\varepsilon(k) \in\left(0, C_{4} / 2(k T)^{p}\right)$; then from (3.24), we have

$$
\varphi_{k}(u) \geq C_{4} \rho_{k}^{p}-k T \varepsilon \delta^{p} \geq \frac{C_{4}}{2} \rho_{k}^{p} \equiv \alpha>0
$$

for all $u \in \widetilde{W}_{T}^{1, p}$ and $\|u\|=\rho_{k}$. This implies that condition (a) of Lemma 2.2 holds. 
Step 3. Let $c=\max \left\{c_{j}\right\}, j \in B$. Choose $C_{7}>\left(c_{2} / p\right)+(m c / T)$; then from (H3), there exists $M_{4}>0$ such that

$$
F(t, x) \geq C_{7}|x|^{p}, \quad|x| \geq M_{4} \text {, a.e. } t \in[0, T] .
$$

By assumption (A), for $|x| \leq M_{4}$, there exists $C_{8}=\max _{|x| \leq M_{4}} a(|x|)>0$ such that

$$
|F(t, x)| \leq C_{8} b(t), \quad \text { a.e. } t \in[0, T],
$$

which together with (3.37) implies that

$$
F(t, x) \geq C_{7}|x|^{p}-C_{8} b(t), \quad \forall x \in \mathbb{R}^{N} \text {, a.e. } t \in[0, T]
$$

Thus, from (H1), (I1), (3.8), and (3.39), we have

$$
\begin{aligned}
\phi_{k}(u) & =\frac{1}{p} \int_{0}^{k T}\left(L(t)|u|^{p-2} u, u\right) d t-\int_{0}^{k T} F(t, u) d t+k \sum_{j=1}^{m} I_{j}(u) \\
& =\frac{k}{p} \int_{0}^{T}\left(L(t)|u|^{p-2} u, u\right) d t-k \int_{0}^{T} F(t, u) d t+k \sum_{j=1}^{m} I_{j}(u) \\
& \leq \frac{c_{2} k}{p} \int_{0}^{T}|u|^{p} d t-k \int_{0}^{T} C_{7}|u|^{p} d t+k \int_{0}^{T} C_{8} b(t) d t+m c|u|^{p} \quad \text { for } u \in \mathbb{R}^{N} .
\end{aligned}
$$

From (H3), we can choose $C_{7}$ suitable large such that

$$
\phi_{k}(u) \leq 0, \quad \forall u \in \mathbb{R}^{N}
$$

Let $\bar{W}_{k T}^{1, p}=\operatorname{span}\left\{e_{k}\right\}+\mathbb{R}^{N}$, where $e_{k}=\left(k^{-1} \sin \left(k^{-1} \omega t\right)\right), \omega=2 \pi / T$. Since $\bar{W}_{T}^{1, p}$ is finite dimensional, there exists a constant $d>0$ such that

$$
\left(\int_{0}^{T}|x|^{p} d t\right)^{1 / p} \geq d\left(\int_{0}^{T}|x|^{2} d t\right)^{1 / 2}, \quad \forall x \in \bar{W}_{T}^{1, p}
$$


Journal of Applied Mathematics

By (I1), we have

$$
\begin{aligned}
\left|\psi\left(u+r e_{k}\right)\right| & =\left|k \sum_{j=1}^{m} I_{j}\left(u+r e_{k}\left(t_{j}\right)\right)\right| \\
& \leq \sum_{j=1}^{m} c_{j}\left|u+r e_{k}\left(t_{j}\right)\right|^{p} \\
& \leq 2^{p} m c|u|^{p}+2^{p} m c r^{p}\left|e_{k}\left(t_{j}\right)\right|^{p} \\
& \leq 2^{p} m c|u|^{p}+2^{p} m c r^{p} k^{-p} \\
& \leq 2^{p} m c|u|^{p}+2^{p} m c r^{p}, \quad u \in \mathbb{R}^{N} .
\end{aligned}
$$

From (3.39), (3.42), and (3.43), we obtain

$$
\begin{aligned}
\phi_{k}\left(u+r e_{k}\right)= & \frac{1}{p} \int_{0}^{k T}\left|r \dot{e}_{k}(t)\right|^{p} d t-\int_{0}^{k T} F\left(t, u+r e_{k}(t)\right) d t \\
& +\frac{1}{p} \int_{0}^{k T}\left(L(t)\left|u+r e_{k}(t)\right|^{p-2}\left(u+r e_{k}(t)\right), u+r e_{k}(t)\right) d t+k \sum_{j=1}^{m} I_{j}\left(u+r e_{k}\left(t_{j}\right)\right) \\
\leq & \frac{1}{p} k^{-2 p} r^{p} \omega^{p} \int_{0}^{k T}\left|\cos \left(k^{-1} \omega t\right)\right|^{p} d t+\frac{c_{2}}{p} \int_{0}^{k T}\left|u+r e_{k}(t)\right|^{p} d t+\int_{0}^{k T} C_{8} b(t) d t \\
& -\int_{0}^{k T} C_{7}\left|u+r e_{k}(t)\right|^{p} d t+2^{p} m c|u|^{p}+2^{p} m c r^{p} \\
\leq & \frac{1}{p} k^{-2 p+1} r^{p} \omega^{p} \int_{0}^{T}|\cos (\omega t)|^{p} d t-k \int_{0}^{T}\left(C_{7}-\frac{c_{2}}{p}\right)\left|u+r e_{1}(t)\right|^{p} d t \\
& +\int_{0}^{T} C_{8} k b(t) d t+2^{p} m c|u|^{p}+2^{p} m c r^{p} \\
\leq & \left(\frac{T}{p} k^{-2 p+1} \omega^{p}+2^{p} m c\right) r^{p}-k d^{p}\left(C_{7}-\frac{c_{2}}{p}\right)\left(\int_{0}^{T}\left|u+r e_{1}(t)\right|^{2} d t\right)^{p / 2} \\
& +2^{p} m c|u|^{p}+C_{9} k \\
\leq & \left(\frac{T}{p} k^{-2 p+1} \omega^{p}+2^{p} m c\right) r^{p}-k d^{p}\left(C_{7}-\frac{c_{2}}{p}\right)\left(\int_{0}^{T}\left(|u|^{2}+r^{2}\left|e_{1}(t)\right|^{2}\right) d t\right) \\
& +2^{p} m c|u|^{p}+C_{9} k \\
\leq & \left(\frac{T}{p} k^{-2 p+1} \omega^{p}+2^{p} m c\right) r^{p}-k d^{p}\left(C_{7}-\frac{c_{2}}{p}\right)\left(T|u|^{2}+\frac{T r^{2}}{2}\right)^{p / 2} \\
& +C_{9} k, \quad \forall r \geq 0, u \in \mathbb{R}^{N} .
\end{aligned}
$$


From (H3), we can choose $C_{7}$ suitable such that

$$
\begin{gathered}
d^{p}\left(C_{7}-\frac{c_{2}}{p}\right)\left(\frac{T}{2}\right)^{p / 2}-2^{3 p} m c>0, \\
d^{p}\left(C_{7}-\frac{c_{2}}{p}\right) T^{p / 2}-2^{p+1} m c>0 .
\end{gathered}
$$

If $k \geq 2(T p)^{1 / 2 p} \omega^{1 / 2} /\left[d^{p}\left(C_{7}-c_{2} / p\right)(T / 2)^{p / 2}-2^{3 p} m c\right]:=C_{10}$, then we get

$$
\begin{aligned}
k^{-1} \phi_{k}\left(u+r e_{k}\right) & \leq\left[\frac{T}{p} k^{-2 p} r^{p} \omega^{p}+\frac{2^{p} m c}{k}-d^{p}\left(C_{7}-\frac{c_{2}}{p}\right)\left(\frac{T}{2}\right)^{p / 2}\right] r^{p}+C_{9} \\
& \leq\left[T k^{-2 p} \omega^{p}+2^{p} m c-d^{p}\left(C_{7}-\frac{c_{2}}{p}\right)\left(\frac{T}{2}\right)^{p / 2}\right] r^{p}+C_{9} \\
& \leq-\frac{1}{2} d^{p}\left(C_{7}-\frac{c_{2}}{p}\right)\left(\frac{T}{2}\right)^{p / 2} r^{p}+C_{9}, \\
k^{-1} \phi_{k}\left(u+r e_{k}\right) & \leq-\frac{1}{2} d^{p}\left(C_{7}-\frac{c_{2}}{p}\right) T^{p / 2}|u|^{p}+C_{9} .
\end{aligned}
$$

It follows from (3.46) that

$$
\varphi_{k}\left(u+r e_{k}\right) \leq 0, \quad \text { either } r \geq r_{1} \text { or }|u| \geq r_{2}
$$

where $r_{1}=\sqrt{2}\left(2 C_{9}\right)^{1 / p} /\left(C_{7}-c_{2} / p\right)^{1 / p} d T^{1 / 2}, r_{2}=\left(2 C_{9}\right)^{1 / p} / d\left(C_{7}-c_{2} / p\right)^{1 / p} T^{1 / 2}$. Notice that for any $u \in \mathbb{R}^{N}$, we have

$$
\|u\|=\|u\|_{L^{p}}=\left(\int_{0}^{k T}|u|^{p} d t\right)^{1 / p}=(k T)^{1 / p}|u| \geq\left(C_{10} T\right)^{1 / p} r_{2}:=r_{3} .
$$

Hence, (3.47) holds for all $\|u\| \geq r_{3}$ whenever $u \in \mathbb{R}^{N}$. Set

$$
Q_{k}=\left\{r e_{k} \mid 0 \leq r \leq r_{1}, e_{k} \in \widetilde{W}_{k T}^{1, p}\right\} \oplus\left\{u \in \mathbb{R}^{N} \mid\|u\| \leq r_{3}\right\}
$$

then $\partial Q_{k}=Q_{1 k} \cup Q_{2 k} \cup Q_{3 k}$, where

$$
\begin{aligned}
& Q_{1 k}=\left\{u \in \mathbb{R}^{N} \mid\|u\| \leq r_{3}\right\}, \\
& Q_{2 k}=\left\{u+r e_{k} \mid\|u\|=r_{3}, r \in\left[0, r_{1}\right], e_{k} \in \widetilde{W}_{k T}^{1, p}\right\}, \\
& Q_{3 k}=\left\{u+r e_{k} \mid\|u\| \leq r_{3}, r=r_{1}, e_{k} \in \widetilde{W}_{k T}^{1, p}\right\} .
\end{aligned}
$$


By (3.41) and (3.47), we have

$$
\varphi(u) \leq 0, \quad u \in \partial Q_{k}=Q_{1 k} \bigcup Q_{2 k} \bigcup Q_{3 k} .
$$

Furthermore, for all $u+r e_{k} \in Q_{k}$, it follows from (H1), (3.8), and (3.43) that

$$
\begin{aligned}
\phi_{k}\left(u+r e_{k}\right)= & \frac{1}{p} \int_{0}^{k T}\left|r \dot{e}_{k}(t)\right|^{p} d t-\int_{0}^{k T} F\left(t, u+r e_{k}(t)\right) d t \\
& +\frac{1}{p} \int_{0}^{k T}\left(L(t)\left|u+r e_{k}(t)\right|^{p-2}\left(u+r e_{k}(t)\right), u+r e_{k}(t)\right) d t+k \sum_{j=1}^{m} I_{j}\left(u+r e_{k}\left(t_{j}\right)\right) \\
\leq & \frac{1}{p} r^{p} \int_{0}^{k T}\left|\dot{e}_{k}(t)\right|^{p} d t+\frac{c_{2}}{p} \int_{0}^{k T}\left|u+r e_{k}(t)\right|^{p} d t+2^{p} m c|u|^{p}+2^{p} m c r^{p} \\
\leq & \frac{1}{p} k^{-2 p} r^{p} \omega^{p} \int_{0}^{k T}\left|\cos \left(k^{-1} \omega t\right)\right|^{p} d t+\frac{2^{p-1} c_{2}}{p} \int_{0}^{k T}\left(|u|^{p}+r^{p} k^{-p}\left|\sin \left(k^{-1} \omega t\right)\right|^{p}\right) d t \\
& +2^{p} m c|u|^{p}+2^{p} m c r^{p} \\
\leq & \frac{1}{p} k^{-2 p+1} r^{p} \omega^{p} \int_{0}^{T}|\cos (\omega t)|^{p} d t+\frac{2^{p-1} c_{2}}{p}\left(\|u\|^{p}+r^{p} k^{-p+1} \int_{0}^{T}|\sin (\omega t)|^{p} d t\right) \\
& +\frac{2^{p} m c}{T}\|u\|^{p}+2^{p} m c r^{p} \\
\leq & \frac{T}{p} k^{-2 p+1} r^{p} \omega^{p}+\frac{2^{p-1} c_{2}}{p}\left(\|u\|^{p}+r^{p} k^{-p+1} T\right)+\frac{2^{p} m c}{T}\|u\|^{p}+2^{p} m c r^{p} \\
\leq & \frac{T}{p} r_{1}^{p} \omega^{p}+\frac{2^{p-1} c_{2}}{p}\left(r_{3}^{p}+r_{1}^{p} T\right)+2^{p} m c\left(\frac{1}{T} r_{3}^{p}+r_{1}^{p}\right) .
\end{aligned}
$$

Then by Lemma $2.2, \phi_{k}$ has at least a critical point $u_{k}$ whose critical value $c_{k}$ satisfies

$$
0<\alpha \leq c_{k}=\phi_{k}\left(u_{k}\right) \leq \frac{T}{p} r_{1}^{p} \omega^{p}+\frac{2^{p-1} c_{2}}{p}\left(r_{3}^{p}+r_{1}^{p} T\right)+2^{p} m c\left(\frac{1}{T} r_{3}^{p}+r_{1}^{p}\right) .
$$

Similar to the proof of [28], let $u_{k_{1}}$ be a $k_{1} T$-periodic solution; we can prove that there exists a positive integer $k_{2}>k_{1}$ such that $u_{k k_{1}} \neq u_{k_{1}}$ for all $k k_{1} \geq k_{2}$. Otherwise, $\varphi_{k}\left(u_{k k_{1}}\right)=$ $k \varphi_{k}\left(u_{k_{1}}\right) \rightarrow \infty$ as $k \rightarrow \infty$, which contradicts to (3.53). Repeating this process, we can obtain a sequence $\left\{u_{k_{j}}\right\}$ of distinct periodic solutions of problem (1.1). From (3.41), we know that $u_{k_{j}}$ is nonconstant. The proof is complete.

\section{Examples}

In this section, we give an example to illustrate our result. 
Example 4.1. Let $p=3, r=5, \mu=4$, and consider the following $p$-Laplacian system with impulsive effects

$$
\begin{gathered}
\frac{d}{d t}(|\dot{u}(t)| \dot{u}(t))-L(t)|u(t)| u(t)+\nabla F(t, u(t))=0, \quad \text { a.e. } t \in \mathbb{R}, \\
u(0)-u(T)=\dot{u}(0)-\dot{u}(T)=0, \\
\Delta\left(\left|\dot{u}\left(t_{i}\right)\right| \dot{u}\left(t_{i}\right)\right)=\left|\dot{u}\left(t_{i}^{+}\right)\right| \dot{u}\left(t_{i}^{+}\right)-\left|\dot{u}\left(t_{i}^{-}\right)\right| \dot{u}\left(t_{i}^{-}\right)=\nabla I_{i}\left(u\left(t_{i}\right)\right), \quad i=1,2, \ldots, m .
\end{gathered}
$$

Let

$$
\begin{gathered}
L(t)=\operatorname{diag}\left(1+\exp \left(1-\sin \left(k^{-1} \omega t\right)\right), \ldots, 1+\exp \left(1-\sin \left(k^{-1} \omega t\right)\right)\right), \\
I_{i}(x)=\frac{c_{i}}{k}|x|^{p}, \quad F(t, x)=\frac{1+e}{3}\left(2+\sin \left(k^{-1} \omega t\right)\right)|x|^{5},
\end{gathered}
$$

where $c_{i}>0, i \in B$. It is easy to check that $F$ satisfies (A), (H1), and (H2). By a direct computation, we have

$$
\begin{gathered}
\lim _{|x| \rightarrow \infty} \frac{F(t, x)}{|x|^{3}}=+\infty, \quad \limsup _{|x| \rightarrow \infty} \frac{F(t, x)}{|x|^{5}} \leq 1+e, \\
\liminf _{|x| \rightarrow \infty} \frac{(\nabla F(t, x), x)-3 F(t, x)}{|x|^{4}} \geq \frac{2(1+e)}{3},
\end{gathered}
$$

which show that (H3), (H4), and (H5) hold. On the other hand,

$$
0 \leq I_{i}(x) \leq \frac{c}{k}|x|^{p}, \quad \nabla I_{i}(x) x=\frac{p c_{i}}{k}|x|^{p}=p I_{i}(x),
$$

where $c=\max \left\{c_{i}\right\}, i \in B$. It is easy to see that $I_{i}$ satisfies (I1) and (I2). Hence, from Theorem 3.1, problem (4.1) has a sequence of distinct nonconstant periodic solutions with period $k_{j} T$ satisfying $k_{j} \in \mathbb{N}$ and $k_{j} \rightarrow \infty$ as $j \rightarrow \infty$.

\section{Acknowledgments}

W.-Z. Gong is supported by Guangxi Natural Science Foundation (2010GXNSFA013125) and X. H. Tang is supported by the NNSF (no. 10771215) of China.

\section{References}

[1] Y. H. Ding, "Existence and multiplicity results for homoclinic solutions to a class of Hamiltonian systems," Nonlinear Analysis. Theory, Methods \& Applications, vol. 25, no. 11, pp. 1095-1113, 1995.

[2] Y. Ding and C. Lee, "Homoclinics for asymptotically quadratic and superquadratic Hamiltonian systems," Nonlinear Analysis. Theory, Methods \& Applications, vol. 71, no. 5-6, pp. 1395-1413, 2009. 
[3] X. He and X. Wu, "Periodic solutions for a class of nonautonomous second order Hamiltonian systems," Journal of Mathematical Analysis and Applications, vol. 341, no. 2, pp. 1354-1364, 2008.

[4] Z.-Q. Ou and C.-L. Tang, "Existence of homoclinic solution for the second order Hamiltonian systems," Journal of Mathematical Analysis and Applications, vol. 291, no. 1, pp. 203-213, 2004.

[5] J. Wang, F. Zhang, and J. Xu, "Existence and multiplicity of homoclinic orbits for the second order Hamiltonian systems," Journal of Mathematical Analysis and Applications, vol. 366, no. 2, pp. 569-581, 2010.

[6] Q. Zhang and X. H. Tang, "New existence of periodic solutions for second order non-autonomous Hamiltonian systems," Journal of Mathematical Analysis and Applications, vol. 369, no. 1, pp. 357-367, 2010.

[7] Q. Zhang and C. Liu, "Infinitely many homoclinic solutions for second order Hamiltonian systems," Nonlinear Analysis. Theory, Methods \& Applications, vol. 72, no. 2, pp. 894-903, 2010.

[8] Z. Zhang and R. Yuan, "Homoclinic solutions for a class of non-autonomous subquadratic secondorder Hamiltonian systems," Nonlinear Analysis. Theory, Methods E Applications, vol. 71, no. 9, pp. 4125-4130, 2009.

[9] D. D. Bănov and P. Simeonov, Impulsive Differential Equations: Periodic Solutions and Applications, vol. 66, Longman Scientific \& Technical, Harlow, UK, 1993.

[10] V. Lakshmikantham, D. D. BaĬnov, and P. S. Simeonov, Theory of Impulsive Differential Equations, vol. 6, World Scientific Press, Singapore, 1989.

[11] J. Chu and J. J. Nieto, "Impulsive periodic solutions of first-order singular differential equations," Bulletin of the London Mathematical Society, vol. 40, no. 1, pp. 143-150, 2008.

[12] Y.-K. Chang, J. J. Nieto, and W.-S. Li, “On impulsive hyperbolic differential inclusions with nonlocal initial conditions," Journal of Optimization Theory and Applications, vol. 140, no. 3, pp. 431-442, 2009.

[13] D. Franco and J. J. Nieto, "Nonlinear boundary value problems for first order impulsive functional differential equations," Journal of Computational and Applied Mathematics, vol. 88, pp. 149-159, 1998.

[14] Y. Li, "Positive periodic solutions of nonlinear differential systems with impulses," Nonlinear Analysis. Theory, Methods \& Applications, vol. 68, no. 8, pp. 2389-2405, 2008.

[15] Z. Luo and J. J. Nieto, "New results for the periodic boundary value problem for impulsive integrodifferential equations," Nonlinear Analysis. Theory, Methods \& Applications, vol. 70, no. 6, pp. 2248-2260, 2009.

[16] E. K. Lee and Y.-H. Lee, "Multiple positive solutions of singular two point boundary value problems for second order impulsive differential equations," Applied Mathematics and Computation, vol. 158, no. 3, pp. 745-759, 2004.

[17] J. J. Nieto, "Impulsive resonance periodic problems of first order," Applied Mathematics Letters, vol. 15, no. 4, pp. 489-493, 2002.

[18] J. J. Nieto and R. Rodríguez-López, “Boundary value problems for a class of impulsive functional equations," Computers \& Mathematics with Applications, vol. 55, no. 12, pp. 2715-2731, 2008.

[19] P. Chen and X. H. Tang, "Existence of solutions for a class of p-Laplacian systems with impulsive effects," Journal of Taiwanses Mathematics. In press.

[20] J. J. Nieto and D. O'Regan, "Variational approach to impulsive differential equations," Nonlinear Analysis. Real World Applications, vol. 10, no. 2, pp. 680-690, 2009.

[21] J. Sun, H. Chen, and L. Yang, "The existence and multiplicity of solutions for an impulsive differential equation with two parameters via a variational method," Nonlinear Analysis. Theory, Methods $\mathcal{E}$ Applications, vol. 73, no. 2, pp. 440-449, 2010.

[22] J. Sun, H. Chen, and L. Yang, "Variational methods to fourth-order impulsive differential equations," Journal of Applied Mathematics and Computing, vol. 35, no. 1-2, pp. 323-340, 2011.

[23] Y. Tian and W. Ge, "Applications of variational methods to boundary-value problem for impulsive differential equations," Proceedings of the Edinburgh Mathematical Society. Series II, vol. 51, no. 2, pp. 509-527, 2008.

[24] J. W. Zhou and Y. K. Li, "Existence and multiplicity of solutions for some Dirichlet problems with impulsive effects," Nonlinear Analysis. Theory, Methods \& Applications, vol. 71, no. 7-8, pp. 2856-2865, 2009.

[25] J. Zhou and Y. Li, "Existence of solutions for a class of second-order Hamiltonian systems with impulsive effects," Nonlinear Analysis. Theory, Methods \& Applications, vol. 72, no. 3-4, pp. 1594-1603, 2010.

[26] J. Mawhin and M. Willem, Critical Point Theory and Hamiltonian Systems, vol. 74, Springer, New York, NY, USA, 1989. 
[27] P. H. Rabinowitz, Minimax Methods in Critical Point Theory with Applications to Differential Equations, vol. 65 of CBMS Regional Conference Series in Mathematics, American Mathematical Society, Providence, RI, USA, 1986.

[28] S. W. Ma and Y. X. Zhang, "Existence of infinitely many periodic solutions for ordinary p-Laplacian systems," Journal of Mathematical Analysis and Applications, vol. 351, no. 1, pp. 469-479, 2009. 


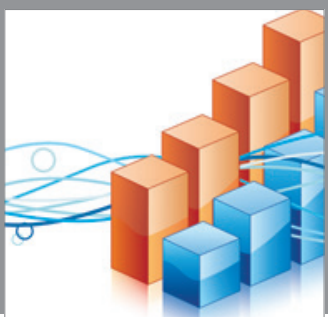

Advances in

Operations Research

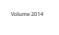

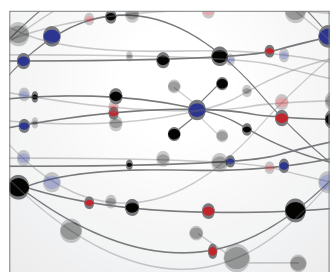

\section{The Scientific} World Journal
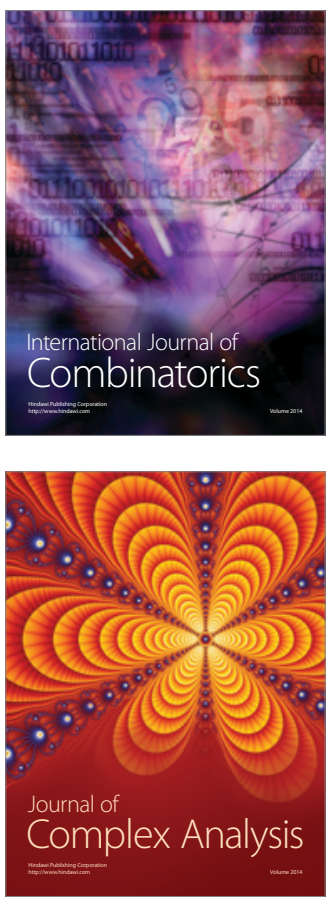

International Journal of

Mathematics and

Mathematical

Sciences
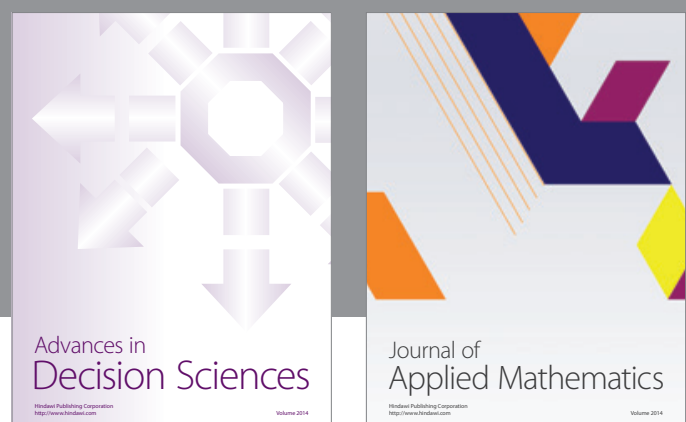

Journal of

Applied Mathematics
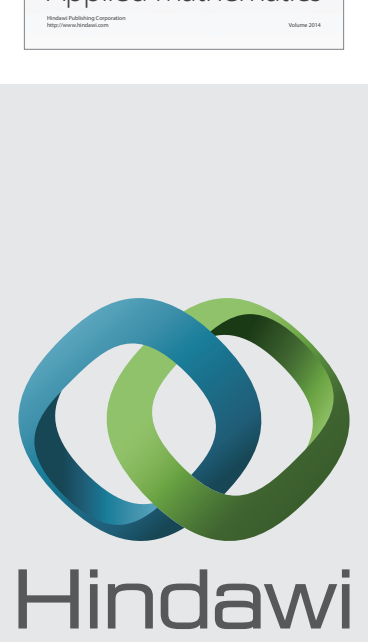

Submit your manuscripts at http://www.hindawi.com
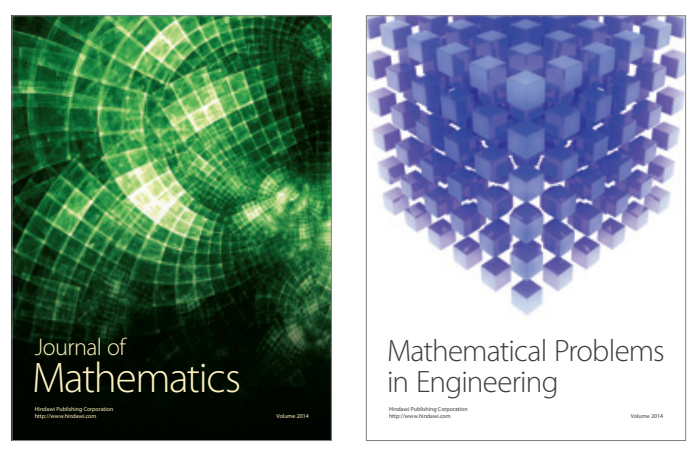

Mathematical Problems in Engineering
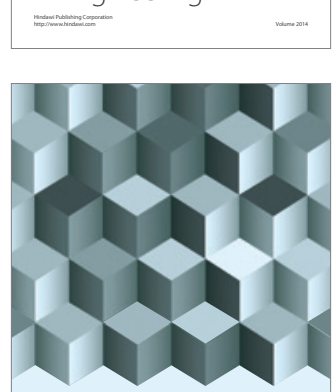

Journal of

Function Spaces
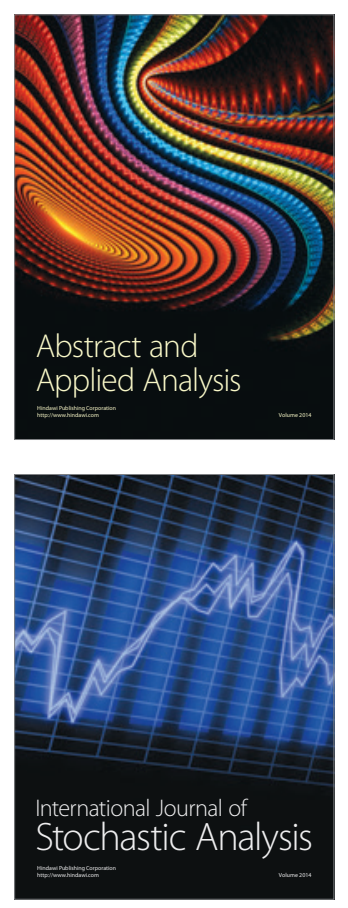

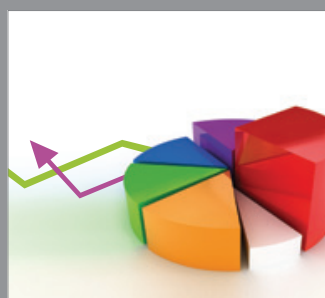

ournal of

Probability and Statistics

Promensencen
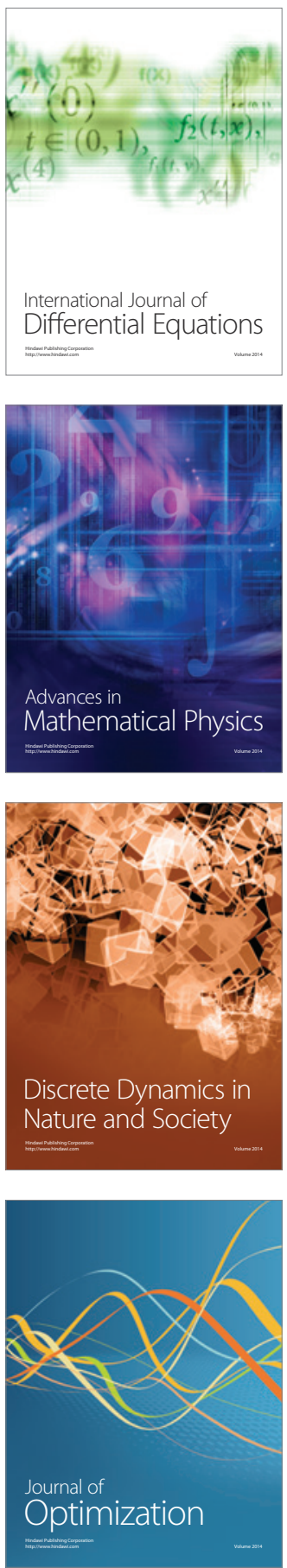